\title{
Brain regional homogeneity and function connectivity in attenuated psychosis syndrome - based on a resting state fMRI study
}

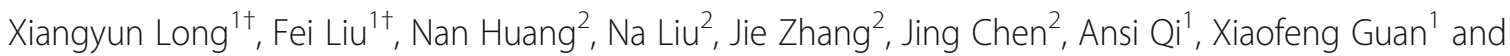
Zheng $L u^{1,2^{*}}$

\begin{abstract}
Background: By combining regional homogeneity (ReHo) and functional connectivity (FC) analyses, this study aimed to explore brain functional alterations in Attenuated Psychosis Syndrome (APS), which could provide complementary information for the neurophysiological indicators for schizophrenia (SZ) associated brain dysfunction.

Methods: Twenty-one APS subjects and twenty healthy controls were enrolled in the data acquisition of demographics and clinical characteristics as well as structural and resting-state functional magnetic resonance imaging (rs-fMRI). ReHo analysis was conducted to determine the peak coordinate of the abnormal regional brain activity. Then, identified brain regions were considered as seed regions and were used to calculate FC between reginal brain voxels and whole brain voxels. Finally, potential correlations between imaging indices and clinical data were also explored.

Results: Four APS and two HC subjects were excluded because the largest dynamic translation or rotation had exceeded $2 \mathrm{~mm} / 2^{\circ}$. Compared with healthy controls (HCs), APS subjects exhibited higher ReHo values in the right middle temporal gyrus (MTG) and lower ReHo values in the left middle frontal gyrus (MFG), left superior frontal gyrus (SFG), left postcentral gyrus (PoCG), and left superior frontal gyrus, medial (SFGmed). Considered these areas as seed regions, the APS subjects showed abnormal enhancement in functional brain connections, predominantly in the frontal and temporal lobes.
\end{abstract}

Conclusions: We concluded that the APS subjects had spatially regional dysfunction and remoted synchronous dysfunction in the frontal and temporal lobes of the brain, and changes in ReHo and FC patterns may reveal the mechanism of brain dysfunctions and may serve as an imaging biomarker for the diagnosis and evaluation of SZ.

Keywords: Attenuated psychosis syndrome, Resting-state functional magnetic resonance imaging, Regional homogeneity, Functional connectivity

\footnotetext{
* Correspondence: luzheng@tongji.edu.cn

${ }^{+}$Xiangyun Long and Fei Liu contributed equally to this work.

'Department of Psychiatry, Shanghai Tongji Hospital, Tongji University School

of Medicine, 389 Xin Cun Road, Shanghai 200065, China

${ }^{2}$ Department of Psychiatry, Shanghai Mental Health Center, Shanghai Jiao

Tong University School of Medicine, 600 Wan Ping Nan Road, Shanghai

200030, China
}

(c) The Author(s). 2018 Open Access This article is distributed under the terms of the Creative Commons Attribution 4.0 International License (http://creativecommons.org/licenses/by/4.0/), which permits unrestricted use, distribution, and reproduction in any medium, provided you give appropriate credit to the original author(s) and the source, provide a link to the Creative Commons license, and indicate if changes were made. The Creative Commons Public Domain Dedication waiver (http://creativecommons.org/publicdomain/zero/1.0/) applies to the data made available in this article, unless otherwise stated. 


\section{Background}

Seventy to ninety percent of schizophrenic patients would experience behavioral, verbal, and cognitive abnormalities before they reach the diagnosis of schizophrenia [1, 2]. Some researchers named this phase as Attenuated Psychosis Syndrome (APS), and this diagnostic term was also included in the section III of the Diagnostic and Statistical Manual of Mental Disorders, Fifth Edition (DSM-5) [3, 4]. The APS was also called "UHR (Ultra High Risk)" "ARMS (At Risk Mental State)" or "PRS (Psychosis Risk Syndrome)", etc. [5], and it propelled scientists to research and develop preventions, identifications, and intervention programs for early psychosis. This definition helped us to screen out individuals with psychotic symptoms and need clinical interventions [6, 7]. Individuals with APS were considered to be highly at risk to psychosis: about $20-25 \%$ of them would convert to psychosis within 1 year and 30 $35 \%$ within 2 years [8]. It is a well-accepted fact that even though schizophrenia (SZ) was classified as a severe mental illness, we don't have much to serve as qualitative or quantitative biological indicators; its etiological research involved not only schizophrenic patients, but also included APS, first-degree relatives and other related groups. Previous studies were able to discover some symptomatology predictors in the APS group, including impairments in working memory, general intelligence, executive functions, verbal/visual memory, verbal fluency, attention and social cognition $[9,10]$ And since the functional magnetic resonance imaging (fMRI) was applied in this research, it became the excellent tool in exploring brain dysfunctions under mental symptoms.

Previous studies of APS neuroimaging were able to cover the resting-state functional magnetic resonance imaging (fMRI) and the task fMRI, such as discoveries in the prefrontal cortex (PFC) [8] and the white matter damage [11]. These structural anomalies propelled functional researches. Functional changes were often discussed along with changes in cognitive functions, language network dysfunctions [12], and activation anomalies in anterior cingulate cortex (ACC) [13] and bilateral PFC [14]. Social impairments had a negative correlation with anomalies in basal orbitofrontal cortex [15]. The combined results of functional and structural images seemed to be more precise in predicting APS subjects while comparing with results of unilateral image. Tognin used the method of multivariate machine learning to predict clinical symptoms and brain structure anomalies in APS subjects by gray matter volume and cortical thickness [16]. Benetti \& Williams had compared genetic, cognitive and multi modal neuroimaging data to identify APS, first-episode psychosis (FEP) and healthy controls (HCs), and got $68.42 \%$ successful classification accuracies with structural magnetic resonance imaging and $65.79 \%$ with diffusion tensor neuroimaging in identifying APS subjects from healthy controls [17]. These results provided us with an inspiration, which is to analyze neuroimages of APS subjects.

It has been proved that organization functional brain networks develop from an anatomically local status to a more distributed structure [18]. In previous schizophrenia researches, a few guidelines had been established by studying regional and global brain function's synchronization. Individuals identified as APS may be at a higher risk of developing schizophrenia than general population. Previous studies related to functional connectivity (FC) had also shown that this higher risk may have something to do with the unequal connection between ACC [13], ventral striatum [19], thalamus [20], bilateral frontal gyrus and other whole brain functional connections [21]. But the consistency of the study was not adequate enough due to recruitment of testers, different areas of interest, and analytical methods. And with the progress of methods and examination equipment, current results might point us a way to the disease pattern of psychosis. Some researches on APS made some progress in abnormal regional brain functions and brain synchronizations, which inspired us to embark on our study.

The aim of this article is to use function connectivity (FC) to seek brain function abnormities in APS subjects while compared to HCs. FC was operationally defined as statistical dependencies among distinct neurophysiological events, which can be measured by calculating correlations between time series of voxels or regional seeds, or both $[22,23]$. In order to examine brain dysfunction areas preliminary, we used regional homogeneity (ReHo) to analyze brain function in resting state between groups, it can reflect the consistencies of local brain neurons activities based on calculating Kendall's coefficient concordance (KCC) [24]. We introduced structured Interview for Prodromal Syndromes (SIPS) to evaluate participants for the attenuated psychosis syndrome, which has been proved to quantitatively assess the severity of prodromal symptoms for psychosis [25].

\section{Methods \\ Participants}

Twenty-one subjects with APS and 20 healthy controls (HCs) were recruited from studies of SZ early diagnosis between Feb 2014 and Dec 2014. All of our participants were outpatients from Shanghai Tongji Hospital and Shanghai Mental Health Center (SMHC) in China, and were all assessed by trained doctors. The ethical protocol was approved by Tongji hospital and SMHC, carried out in accordance with the Declaration of Helsinki. We ensured that all participants were informed of the study.

This study used the Chinese version SIPS, translated by Zheng [24], to evaluate the prodromal symptoms of APS subjects. SIPS is a structured interviewing tool 
based on SOPS (Scale of Psychosis-risk Symptoms), the full assessment takes about $1 \mathrm{~h}$, and other scales including GAF (Global Assessment of Functioning scale), Schizotypal personality disorder checklist and a family history questionnaire [26, 27]. The purpose of using SIPS was to distinguish three prodromal syndromes defined by COPS (Criteria of Prodromal Syndromes) and psychosis onset defined by POPS (Presence of Psychotic Syndrome) [26, 27]. APS subjects must meet at least one category of COPS, and required to be $18-30$ years old, Han nationality, right-handed, untreated with any antipsychotics and Wechsler Intelligence Scale's value $>70$.

HCs were recruited from volunteers and hospital staff and were matched to the participants' age, gender, ethnicity, and educational level with the APS group. We used DSM-IV axis I disorder Structured Clinical Interview for Diagnostic (SCID assessment form) to exclude family history of mental illness or comorbidity.

We set the same exclusion criteria for both groups of subjects, including the inability to receive MRI, family history of mental illness, history of brain trauma or abnormal brain structures display by MRI, and history of alcohol or drug abuse. Demographic data, Positive and Negative Symptom Scale (PANSS) were used to assess their symptoms before performing the fMRI scan [28]. This process was carried out by two experienced psychiatrists who had the experience of 10 years at least from the SMHC.

\section{Image acquisition and preprocessing}

SMHC undertook MRI scans of all subjects, A $3.0 \mathrm{~T}$ MRI scanner with an orthonormal coil (Siemens Magnetom Verio 3.0) was used to obtain a whole-brain T2*weighted echo plane image. All subjects were asked to lie down with their eyes closed, stay awake, and keep their heads still. And then they accepted a consistent scanning process in a time of approximately $6 \mathrm{~min}$ and $40 \mathrm{~s}$, included acquire high-resolution anatomical T1 images (parameters: TR (time to repetition) of $2300 \mathrm{~ms}$, TE (time to echo) of $2960 \mathrm{~ms}$, flip angle of $90^{\circ}$, FOV (field of view) of $256 \mathrm{~mm}$ in $256 \times 256$ matrix, slices thickness of $1.0 \mathrm{~mm}$ ), and a total of 200 functional volumes (parameters: the same orientation as T1 images, in $3 \mathrm{~mm}$ voxel size with a TR of $2000 \mathrm{~ms}$, TE of $30 \mathrm{~ms}$, flip angle of $90^{\circ}$ in $64 \times 64$ matrix size, and $220 \mathrm{~mm}$ FOV), continuous scanning 33 slices with slices thickness of $4.0 \mathrm{~mm}$.

Preprocessing: The functional data preprocess complied with following steps:(1) Format conversion;(2) Remove the previous 10 unstable time points, leaving the remaining 190 time points;(3) Make slice timing using midpoint of time as reference;(4) Realign: set head motion via rigid-body alignment of each volume to the 17 scan, and four APS and two HC subjects were excluded because their largest dynamic rotation or translation had exceeded $2 \mathrm{~mm} / 2^{\circ}$;(5) Specialization of statistical parameters mapped echo-plane Image template in Montreal Institute of Neurology (MNI) Space used a nonlinear warping algorithm and the voxel were resampled to a $3 \mathrm{~mm} * 3$ $\mathrm{mm} * 3 \mathrm{~mm}$ volume unit in a bounding box $(-90-126$ -72;90 90,108);(6) Remove head movement parameters, white matter, cerebrospinal fluid signals and linear drift as covariates; (7) Filter $(0.01<\mathrm{f}<0.08 \mathrm{~Hz})$. Used Resting-State fMRI Data Analysis Toolkit version 1.8(DPARSF Advanced Edition, http://rfmri.org/DPARSF) which was run in Matlab version 7.14(MathWorks R2012a) to conduct.

\section{$F C$ analysis.}

ReHo calculations were performed using preprocessed images, and resulting images were smoothened with an isotropic Gaussian kernel of $4 \mathrm{~mm}$ full-width half- maximum (FWHM).

As mentioned above, we used ReHo analysis to seek the region of interest (ROI) which was calculated using DPARSF after preprocessing. Twenty-seven individual voxels were selected as a mass, using KCC (range 0 to 1 ) as indicators to measure ReHo value between each 27 individual voxels and 26 adjacent voxels. Each individual voxels ReHo was divided by the whole brain average ReHo, then the Gaussian smoothing was proceeded with Full Width Half Width (FWHW) $6 \mathrm{~mm}$ * $6 \mathrm{~mm} " 6 \mathrm{~mm}$ to reduce space noise and error after space standards were produced. Then ReHo differences between the APS and HC group were analyzed with Two-Sample-T-test in Resting-State fMRI Data Analysis Toolkit (REST version1.8, http://pub.restfmri.net/) [29], and were further corrected by monte-carlo simulation (Alphasim correction).

FC calculation was conducted with REST. The coordinate of the center of seed regions was based on the inter-group analysis of ReHo, and its radius was $6 \mathrm{~mm}$. Correlation analysis of time course between the spherical seed area and the whole brain voxel was conducted on each subject. Finally, Fisher's r-to-z transformation was applied to improve the normality of the ReHo and FC maps.

\section{Statistical analysis}

Clinical data and neuropsychological test scores of APS and $\mathrm{HC}$ groups were compared using the SPSS software (version22.0,). Intra-group differences of ReHo and FC were analyzed with one-sample t-test, and inter-group differences between APS and HCs were analyzed with two-sample t-test in REST. The following results were corrected using Monte-Carlo simulation in rest with the program Alphasim, set the statistical threshold of $p<0.001$ (Alphasim correction:cluster $p<0.001$, cluster size $>13$ voxels, full width at half maximum $=6 \mathrm{~mm}$, number of Monte Carlo simulations $=1000$, cluster connection radius: $\mathrm{rmm}=5.00)$ to detect and compare brain regions that survived. 


\section{Results}

Demographics and clinical characteristics of the APS group and the HC group

Eventually, after excluding subjects with excessive head movements, 17 APS subjects and 18 HC subjects entered the statistical analysis stage. Compared with the control group, the APS group had no significant difference between age, education, gender, and body mass index (BMI). (Complete information in Table 1).

\section{Regional homogeneity analysis}

Compared with control group, APS group exhibited significantly higher ReHo values in the right middle temporal gyrus (MTG) and lower ReHo values in the left superior frontal gyrus (SFG), left postcentral gyrus (PoCG), left middle frontal gyrus (MFG), left superior frontal gyrus, medial (SFGmed). (Complete information in Table 2 and Fig. 1).

\section{Functional connectivity analysis}

Compared with the control group, FC between left MFG and right inferior frontal gyrus (IFG), and FC between right MTG to right IFG were increased in the APS group. no positive results were found in the use of other seed points including the left SFG, left PoCG and left SFGmed. (complete information in Table 3 and Fig. 2). Considering with ReHo results, the APS subjects showed abnormally enhanced functional brain connections, predominantly in the frontal and temporal lobes.

\section{Discussion}

Many previous studies have shown the superiority of using fMRI to detect brain dysfunctions among schizophrenic patients, the research's direction had transferred from structural isomerization to single regional dysfunction and now focused on functional connection differences in multiple regions, which refers to the correlation of simultaneous neural activities between the anatomically separated brain regions [30]. Based on the hypothesis that separated brain regions worked together in a stable functional network and maintained a high level sustained spontaneous neuronal activity in resting states, functional connectivity methods were used to explore the pattern and abnormality of interregional functions [31]. Along with the theory above, Some functional sub-networks had been proposed and validated, and these results pushed the fMRI research to be one of the hotspots of mental illness research [32].

By analyzing different functional connectivity between the frequency range of $0.06-0.125 \mathrm{~Hz}$ among $15 \mathrm{HCs}$ and $12 \mathrm{SZ}$ patients, the resting-state fMRI study found that the functional connectivity of SZ patients were significantly reduced but the diversity increased [33]. By utilizing ICA topological measures to analyze the reststate functional network connections in 19 patients, Qingbao Yu discovered patterns of functional connectivity in some regions including frontal, parietal, occipital and cerebellar in SZ group had changed. [19]. In the intra cortical connections, many previous researches focused on abnormal connections of the prefrontal cortex.

Compared to the HCs, APS subjects in this article showed a significantly lower ReHo in left SFG, SFGmed, PoCG, MFG, and a higher ReHo in the right MTG during the resting state. Further FC analysis showed aberrant functional connectivity in the temporal gyrus and the frontal lobe. These abnormalities suggested that it is highly possible that the APS subjects suffered from the disease, which could lead to brain dysfunction from local to global scales. Results of our study were mainly focused on the abnormalities of the frontal and temporal lobes and will be discussed later in the article.

Table 1 Demographics and Clinical Characteristics of the APS Group and the HC Group $(\bar{x} \pm s)$

\begin{tabular}{|c|c|c|c|c|}
\hline & $\operatorname{APS}(n=17)$ & $\mathrm{HC}(n=18)$ & $t / X^{2}$ & $p$ \\
\hline Age (years) & $22.94 \pm 3.39$ & $25.22 \pm 3.68$ & -1.987 & .054 \\
\hline Education level (years) & $13.18 \pm 1.84$ & $12.78 \pm 2.26$ & .565 & .576 \\
\hline BMI (kg/m2) & $20.58 \pm 1.88$ & $21.15 \pm 1.70$ & -.953 & .347 \\
\hline Gender, Male(\%) & $8(47.06)$ & $8(44.44)$ & .024 & .573 \\
\hline \multicolumn{5}{|l|}{ PANSS } \\
\hline Positive & $13.18 \pm 1.19$ & & & \\
\hline Negative & $12.18 \pm 3.59$ & & & \\
\hline General Pychopathology & $24.47 \pm 2.37$ & & & \\
\hline GAF score & $57.05 \pm 3.63$ & & & \\
\hline Family History of Psychosis Disorder, n (\%) & $5(27.77)$ & & & \\
\hline \multicolumn{5}{|l|}{ Primary SIPS-defined prodromal status, n (\%) } \\
\hline Attenuated Positive Symptom State (APSS) & $17(100)$ & & & \\
\hline
\end{tabular}

$\overline{\bar{x}}$ Mean value, s Standard deviation, APS Attenuated psychosis syndrome, HC Healthy controls 
Table 2 Brain regions with different ReHo values between APS and HC groups

\begin{tabular}{|c|c|c|c|c|c|c|c|}
\hline \multirow[t]{2}{*}{ Brain regions } & \multicolumn{3}{|c|}{ MNI:peak voxel } & \multirow{2}{*}{$\begin{array}{l}\text { Cluster } \\
\text { (voxels) }\end{array}$} & \multicolumn{2}{|c|}{ Mean ReHo value( \pm SD) } & \multirow[t]{2}{*}{$t$ value } \\
\hline & $x$ & y & z & & APS & $\mathrm{HC}$ & \\
\hline L Middle frontal gyrus & -33 & -6 & 33 & 61 & $0.85(0.18)$ & $1.27(0.32)$ & -4.395 \\
\hline L Superior frontal gyrus & -18 & 36 & 45 & 20 & $0.90(0.16)$ & $1.17(0.20)$ & -4.010 \\
\hline L Postcentral gyrus & -54 & -12 & 15 & 19 & $0.95(0.20)$ & $1.24(0.27)$ & -4.582 \\
\hline L Superior medial frontal gyrus & -15 & 51 & 12 & 17 & $0.84(0.30)$ & $1.30(0.32)$ & -4.503 \\
\hline R Middle temporal gyrus & 57 & -54 & -6 & 13 & $1.07(0.27)$ & $0.76(0.13)$ & 4.402 \\
\hline
\end{tabular}

APS Attenuated psychosis syndrome, $H C$ Healthy controls, $L$ Left, $R$ Right, MNI Montreal neurological institute, ReHo Regional homogeneity, voxel $p<0.001$ (AlphaSim correction, cluster $p<0.001$, cluster size $>13$ voxels)

The frontal lobe was an important brain region anatomically and functionally, which sub serve human decisionmaking and executive control [34]. Previous studies had divided it into sub-regions based on location and function, including the anterior cingulate, orbital, posterior frontal lobe boundary, etc. A study of neuroanatomical abnormalities in PRS patients found that PRS subjects whom later developed psychosis had lesser grey matter in their inferior frontal gyrus [35], similar findings were also found in SZ subjects [36]. The right inferior frontal gyrus in PRS subjects showed a greater regional functional synchronization in other fMRI researches and showed an increase of function connectivity in our study [37,
38] DTI and task-related fMRI studies found abnormal functional connections in the left and right IFG $[39,40]$. Some studies on schizophrenic patients with drug intervention also points to the variation on structure and function of the frontal lobes [41, 42]. On the whole, our findings on the frontal lobe were consistent with previous studies, which is the importance of frontal lobe in the pathogenesis of SZ.

Previous studies had found that the middle temporal gyrus (MTG) was involved in the default mode (DMN), which was crucial in many cognitive domains such as language processing and deductive reasoning. A crosssectional comparison found that in the right medial

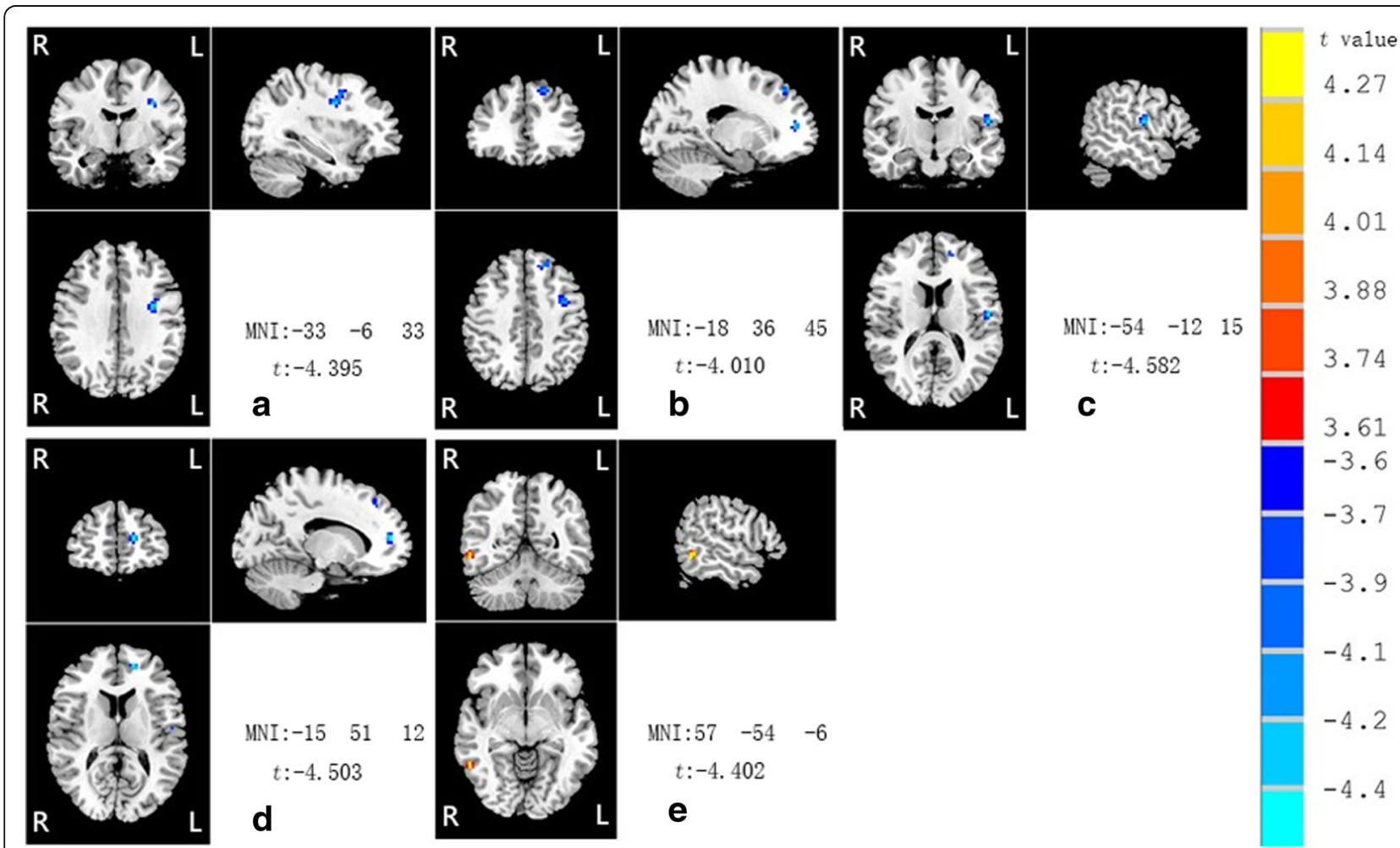

Fig. 1 Compared with control group, APS group exhibited higher ReHo values in the right middle temporal gyrus (MTG) and lower ReHo values in the left middle frontal gyrus (MFG), left superior frontal gyrus (SFG), left postcentral gyrus (PoCG), left superior frontal gyrus, medial (SFGmed) (a left MFG; b left SFG and left SFGmed; c left PoCG and left SFGmed; $\mathbf{d}$ left SFGmed and left SFG; e right MTG) 
Table 3 Brain regions with different FC between APS and HC groups

\begin{tabular}{|c|c|c|c|c|c|c|}
\hline \multirow[t]{2}{*}{ Seed area } & \multirow[t]{2}{*}{ Area with altered functional connectivity } & \multicolumn{3}{|c|}{ MNl:peak voxel } & \multirow{2}{*}{$\begin{array}{l}\text { Cluster } \\
\text { (voxels) }\end{array}$} & \multirow[t]{2}{*}{$t$ value } \\
\hline & & $x$ & y & z & & \\
\hline L Middle Frontal gyrus & R Inferior frontal gyrus & 33 & 3 & 30 & 21 & 4.319 \\
\hline L Superior Frontal gyrus & No significant results & & & & & \\
\hline L Postcentral gyrus & No significant results & & & & & \\
\hline L Superior medial frontal gyrus & No significant results & & & & & \\
\hline R Middle temporal gyrus & R Inferior temporal gyrus/R Middle temporal gyrus & 63 & -42 & -6 & 63 & 5.580 \\
\hline
\end{tabular}

APS Attenuated psychosis syndrome, HC Healthy controls, L Left, $R$ Right, MNI Montreal neurological institute, FC Functional connectivity, voxel $p<0.001$ (Alphasim correction, cluster $p<0.001$, cluster size $>13$ voxels)

temporal area, the volume of gray matter was reduced in PRS subjects who had developed psychosis [43]. And not only PRS subjects had a cortical thinning influenced volumetric reductions of the middle temporal gyrus [35], the same reductions can also be observed among FirstEpisode and Chronic SZ patients studies [44-46]. A study of long-term follow-up of school-age mental illness found that in participates whom developed to SZ, the abnormal decrease of MTG volume preceded the appearance of mental symptoms. [44]. Facial emotion recognition task related MRI also found volume and function changes in temporal lobe in patients with SZ, which may be associated with their impaired facial expression recognition [47]. Studies on brain function differences have confirmed that the temporal gyrus contains multiple sub regions, which was consistent with the results of ReHo and functional synchronization abnormalities in the right temporal gyrus in this study.

There are some restrictions need to be resolved in our future studies. First, we just included a limit number of samples, Most APS populations were children or adolescents, to research on them would have to face multiple limitations such as ethics, schools, families, and so on. And to prepare them for MRI was too difficult and time-consuming, future researches should be focused on improving research projects and designing a more reasonable collection method to overcome these difficulties. Second, patients' compliance with the study wasn't good enough, and their families and schools lacked attention, so we were not able to obtain sufficient follow-up data. Third, for the tools we used in the research, such as SIPS, ReHo, FC method, etc. These templates may not be fully applicable to the Chinese population.

\section{Conclusions}

Our re-fMRI study on the APS population showed that there might be abnormal regional and global brain function synchronization at the resting state. These findings complemented our predecessor's research and the aim were both to explore the pathogenesis of schizophrenia.

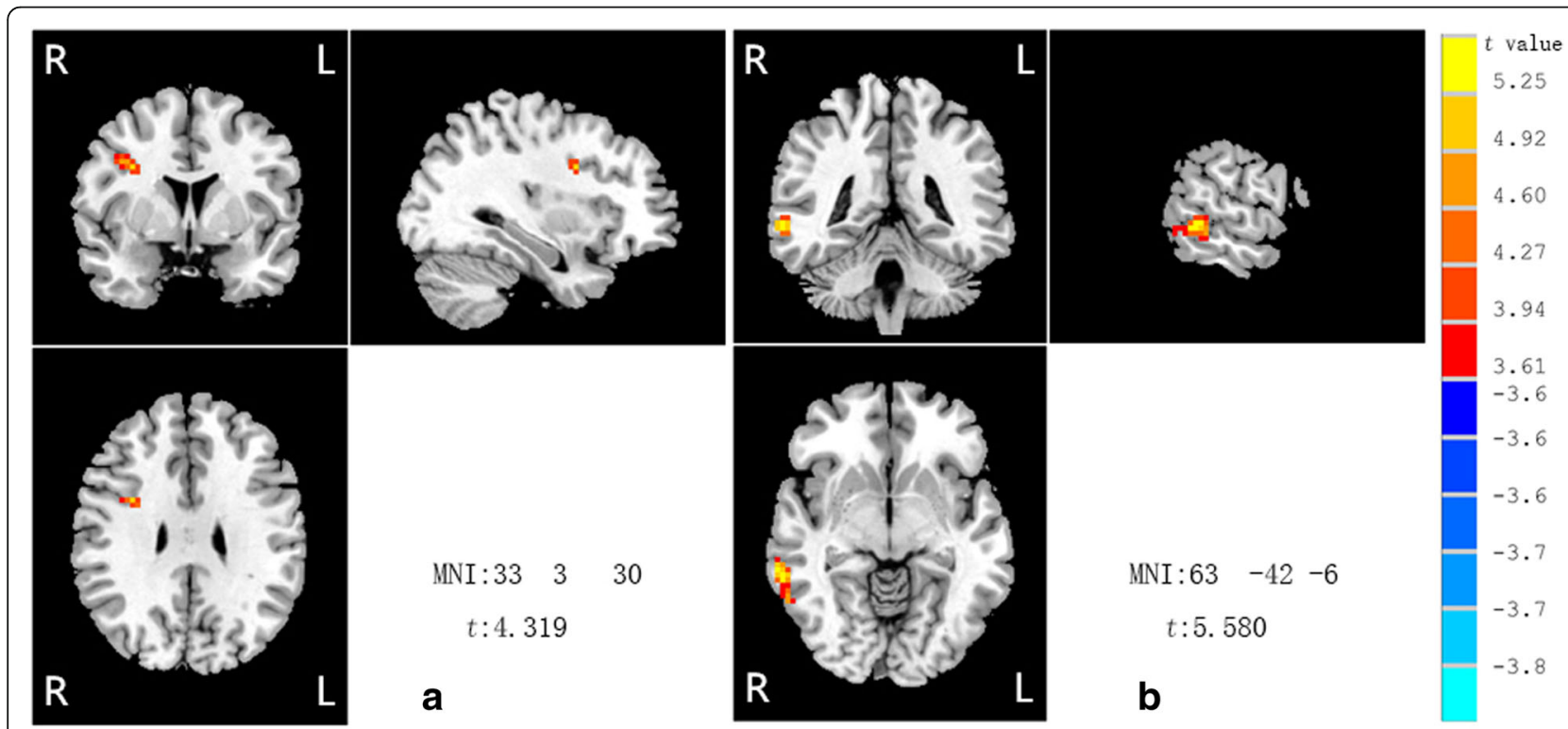

Fig. 2 Compared with the control group, APS group exhibited increased FC between left MFG and right inferior frontal gyrus (IFG), increased FC between right MTG and right IFG (a seed area: left MFG; b seed area: right MTG.) 
We have some train of thought: First, the prodromal phase should be a continuous state; not only the appearance of clinical symptoms, but also the gradual decline of the patient's functions can be served as a starting point of research; Second, it's still unclear whether the abnormal brain function we found in the APS population can directly lead to the appearance of its clinical symptoms; Third, if a model is used to integrate anomaly indicators in multiple dimensions such as brain functional imaging, electroencephalography, physiology, and symptomatology assessments for patients with SZ, can we obtain a more consistent result?

\begin{abstract}
Abbreviations
ACC: Anterior cingulate cortex; APS: Attenuated Psychosis Syndrome; ARMS: At risk mental state; COPS: Criteria of Prodromal Syndromes; DSM5: Diagnostic and Statistical Manual of Mental Disorders, Fifth Edition; DSMIV: Diagnostic and Statistical Manual of Mental Disorders, Fouth Edition: DTI: Diffusion tensorimaging; FC: Functional connectivity; FEP: First-episode psychosis; FOV: Field of view; FWHW: Full-width half- maximum; GAF: Global Assessment of Functioning scale; HC: Healthy control; KCC: Kendall's coefficient concordance; MFG: Middle frontal gyrus; MNI: Montreal Neurological Institute; MTG: Middle temporal gyrus; PANSS: Positive and Negative Symptom Scale; PFC: Prefrontal cortex; PoCG: Postcentral gyrus; POPS: Presence of Psychotic Syndrome; PRS: Psychosis Risk Syndrome; ReHo: Regional homogeneity; ROI: Region of interest; rs-fMRI: Resting-state functional magnetic resonance imaging; SCID: Structured Clinical Interview for Diagnostic; SFG: Superior frontal gyrus; SFGmed: Superior frontal gyrus, medial; SIPS: Structured Interview for Prodromal Syndrome; SMHC: Shanghai Mental Health Center; SOPS: Scale of Psychosis-risk Symptoms; TE: Time to echo; TR: Repetition; UHR: Ultra High Risk
\end{abstract}

\section{Acknowledgements}

We gratefully acknowledged the participation of the individuals involved in the study as well as the valuable and helpful comments of the editors and reviewers on this paper.

\section{Funding}

This work was supported by grants from Priority of Shanghai key discipline of medicine (2017ZZ02020), Ministry of Science and Technology of China (2016YFC1306805), National Natural Science Foundation of China (81471359), Shanghai Science and technology committee (14411963400). The funder had no role in the design, collection, management, analysis, and approval of manuscript or decision to submit the manuscript for publication.

\section{Availability of data and materials}

The dataset(s) generated during the current study are not publically available due to ethical restrictions but are available from the corresponding author on reasonable request.

\section{Authors' contributions}

ZL conceived of the study. XYL, FL and ZL contributed to the design of the study. XYL and FL contributed to the patient recruitment and data acquisition. $\mathrm{XYL}, \mathrm{FL}, \mathrm{NH}, \mathrm{NL}, \mathrm{JZ}$, and JC contributed to the data interpretation and statistical analysis. XYL contributed to the drafting of the paper, ASQ and XFG helped in writing the paper. $F L$ and $Z L$ revised the manuscript critically. All the authors read and approved the final manuscript.

\section{Authors' information}

XYL is PHD student, study at the Department of psychiatry, Tongji Hospital, Tongji University School of Medicine, Shanghai, China. ASQ and XFG are MD student, study at the Department of psychiatry, Tongji Hospital, Tongji University School of Medicine. FL is MD, department of psychiatry, Tongji Hospital, Tongii University School of Medicine. NH, NL, JZ and JC are MD, department of psychiatry, Shanghai Mental Health Center (SMHC), Shanghai Jiao Tong University School of Medicine. ZL is bachelor of medicine, Professor at the Department of psychiatry, Tongji Hospital and SMHC, Shanghai, China.

\section{Ethics approval and consent to participate}

This study was approved by the ethical review committee of Tongji Hospital Affiliated to Tongji Hospital and Shanghai Mental Health Center (SMHC, Shanghai, China), and carried out in accordance with the Declaration of Helsinki. Written informed consent was received from all participants prior to inclusion.

\section{Consent for publication}

Not applicable.

\section{Competing interests}

The authors declare that they have no competing interests.

\section{Publisher's Note}

Springer Nature remains neutral with regard to jurisdictional claims in published maps and institutional affiliations.

Received: 21 May 2018 Accepted: 16 November 2018

Published online: 07 December 2018

\section{References}

1. Yung A, McGorry PD. Prediction of psychosis: setting the stage. Br $\mathrm{J}$ Psychiatry Suppl. 2007;1:1-9.

2. Woods SW, Walsh BC, Saksa JR, Mcglashan TH. The case for including Attenuated Psychotic Symptoms Syndrome in DSM-5 as a psychosis risk syndrome. Schizophr Res. 2011;123:199-207.

3. Schultze-Lutter F, Michel C, Ruhrmann S, Schimmelmann BG. Prevalence and clinical significance of DSM-5-attenuated psychosis syndrome in adolescents and young adults in the general population: the Bern epidemiological at-risk (BEAR) study. Schizophr Bull. 2014;40:1499-508.

4. Carpenter WT, Van Os J. Should attenuated psychosis syndrome be a DSM-5 diagnosis? Am J Psychiatry. 2011;168:460-3.

5. Yung AR, Woods SW, Ruhrmann S, Addington J, Schultze-Lutter F, Cornblatt BA, et al. Whither the attenuated psychosis syndrome? Schizophr Bull. 2012; 38:1130-4.

6. Tsuang MT, Van Os J, Tandon R, Barch DM, Bustillo J, Gaebel W, et al. Attenuated psychosis syndrome in DSM-5. Schizophr Res. 2013;150:31-5. https://doi.org/10.1016/j.schres.2013.05.004 Elsevier B.V.

7. Reddy MS. Attenuated psychosis syndrome. Ind J Psychol Med. 2014;36:1-3.

8. Chung Y, Jacobson A, He G, van Erp TG, McEwen S, Addington J, et al. Prodromal symptom severity predicts accelerated gray matter reduction and third ventricle expansion among clinically high risk youth developing psychotic disorders. Mol Neuropsychiatry. 2015;1:13-22.

9. Yaakub SN, Dorairaj K, Poh JS, Asplund CL, Krishnan R, Lee J, et al. Preserved working memory and altered brain activation in persons at risk for psychosis. Am J Psychiatry. 2013;170:1297-307.

10. Fusar-poli P, Bechdolf A, Taylor MJ, Bonoldi I, Carpenter WT, Yung AR, et al. At risk for schizophrenic or affective psychoses? A Meta-analysis of DSM / ICD diagnostic outcomes in individuals at high clinical risk. Schizophr Bull. 2013;39:923-32

11. Vijayakumar N, Bartholomeusz C, Whitford T, Hermens DF, Nelson B, Rice S, et al. White matter integrity in individuals at ultra-high risk for psychosis: a systematic review and discussion of the role of polyunsaturated fatty acids. BMC Psychiatry. 2016;16:1. https://doi.org/10.1186/s12888-016-0932-4.

12. Sabb FW, van Erp TGM, Hardt ME, Dapretto M, Caplan R, Cannon TD, et al. Language network dysfunction as a predictor of outcome in youth at clinical high risk for psychosis. Schizophr Res. 2010;116:173-83.

13. Lord LD, Allen P, Expert P, Howes O, Broome M, Lambiotte R, et al. Functional brain networks before the onset of psychosis: A prospective fMR study with graph theoretical analysis. Neuroimage Clin. 2012;1:91-8. https:// doi.org/10.1016/j.nicl.2012.09.008.

14. Allen P, Luigjes J, Howes OD, Egerton A, Hirao K, Valli I, et al. Transition to psychosis associated with prefrontal and subcortical dysfunction in ultra high-risk individuals. Schizophr Bull. 2012;38:1268-76

15. Cressman VL, Schobel SA, Steinfeld S, Ben-David S, Thompson JL, Small SA, et al. Anhedonia in the psychosis risk syndrome: associations with social impairment and basal orbitofrontal cortical activity. NPJ Schizophrenia. 2015, 1:15020.

16. Tognin S, Pettersson-Yeo W, Valli I, Hutton C, Woolley J, Allen P, et al. Using structural neuroimaging to make quantitative predictions of symptom 
progression in individuals at ultra-high risk for psychosis. Front Psychiatry. 2014:4:1-9.

17. Benetti S, Williams SCR. Using genetic, cognitive and multi-modal neuroimaging data to identify ultra-high-risk and first-episode; 2015.

18. Liu D, Duan S, Zhang J, Zhou C, Liang M, Yin X, et al. Aberrant Brain Regional Homogeneity and Functional Connectivity in Middle-Aged T2DM Patients: A Resting-State Functional MRI Study. Front Hum Neurosci. 2016;10.

19. Yu Q, Sui J, Rachakonda S, He H, Gruner W, Pearlson G, et al. Altered topological properties of functional network connectivity in schizophrenia during resting state: a small-world brain network study. PLoS One. 2011;6(9):e25423.

20. Anticevic A, Haut K, Murray JD, Repovs G, Yang GJ, Diehl C, et al. Association of thalamic dysconnectivity and conversion to psychosis in youth and young adults at elevated clinical risk. JAMA Psychiatry. 2015; 72:882-91.

21. Wood SJ, Reniers RLEP, Heinze K. Neuroimaging findings in the at-risk mental state : a review of recent neuroimaging findings in the at-risk mental state: a review of recent literature. Can J Psychiatry. 2013;58:13-8.

22. Friston KJ. Functional and effective connectivity: a review. Brain Connect. 2011;1:13-36.

23. Ernst M, Torrisi S, Balderston N, Grillon C, Hale EA. fMRI Functional Connectivity Applied to Adolescent Neurodevelopment. Annu Rev Clin Psychol. 2015;11:361-77.

24. Zang Y, Jiang T, Lu Y, He Y, Tian L. Regional Honogeneity based approach to $\mathrm{fMRI}$ data analysis; 2004

25. Zheng LN, Wang JJ, Zhang TH, Li H, Li CB, Jiang KD. Reliability and validity of the Chinese version of Scale of Psychosis-risk Symptoms. Chin Mental Health J. 2012;26:571-6

26. Miller TJ, McGlashan TH, Rosen JL, Cadenhead K, Ventura J, McFarlane W, et al. Prodromal assessment with the structured interview for prodromal syndromes and the scale of prodromal symptoms: predictive validity, interrater reliability, and training to reliability. Schizophr Bull. 2003;29:703-15.

27. Olsen KA, Rosenbaum B. Prospective investigations of the prodromal state of schizophrenia: assessment instruments. Acta Psychiatr Scand. 2006; 113:273-82

28. Kay SR, Fiszbein A, Opler LA. The positive and negative syndrome scale for schizophrenia. Schizophr Bull. 1987;13:261-76.

29. Song XW, Dong ZY, Long XY, Li SF, Zuo XN, Zhu CZ, et al. REST: a toolkit for resting-state functional magnetic resonance imaging data processing. PLoS One. 2011;6(9):e25031.

30. Friston KJ, Frith CD, Liddle PF, Frackowiak RS. Functional connectivity: the principal-component analysis of large (PET) data sets. J Cereb Blood Flow Metab. 1993;13:5-14.

31. Damoiseaux JS, Greicius MD. Greater than the sum of its parts: a review of studies combining structural connectivity and resting-state functional connectivity. Brain Struct Funct. 2009;213:525-33.

32. van den Heuvel MP, Hulshoff Pol HE. Exploring the brain network: A review on resting-state fMRI functional connectivity. Eur Neuropsychopharmacol. 2010;20:519-34. https://doi.org/10.1016/j.euroneuro.2010.03.008 Elsevier B.V.

33. Lynall M-E, Bassett DS, Kerwin R, McKenna PJ, Kitzbichler M, Muller U, et al. Functional connectivity and brain networks in schizophrenia. J Neurosci. 2010;30:9477-87

34. Collins A, Koechlin E. Reasoning, learning, and creativity: frontal lobe function and human decision-making. PLoS Biol. 2012;10(3):e1001293.

35. Pantelis C, Velakoulis D, Mcgorry PD, Wood SJ, Suckling J, Phillips L, et al. Neuroanatomical abnormalities before and after onset of psychosis: a crosssectional and longitudinal MRI comparison. Lancet. 200;361:281-8.

36. Meisenzahl EM, Koutsouleris N, Gaser C, Bottlender R. Structural brain alterations in subjects at high-risk of psychosis: a voxel-based morphometric study. Schizophr Res. 2008;102:150-62.

37. Howes OD, Allen P, Broome M, Valli I, Asselin M, Montgomery AJ, et al. Abnormal prefrontal activation directly related to pre-synaptic striatal dopamine dysfunction in people at clinical high risk for psychosis. Mol Psychiatry. 2009;16:67-75. https://doi.org/10.1038/mp.2009.108 Nature Publishing Group.

38. Wang S, Wang G, Lv H, Wu R, Zhao J, Guo W. Abnormal regional homogeneity as potential imaging biomarker for psychosis risk syndrome: a resting-state fMRI study and support vector machine analysis. Sci Rep. 2016; 6:1-8. https://doi.org/10.1038/srep27619 Nature Publishing Group.
39. Bleich-Cohen M, Sharon H, Weizman R, Poyurovsky M, Faragian S, Hendler T. Diminished language lateralization in schizophrenia corresponds to impaired inter-hemispheric functional connectivity. Schizophr Res. 2012;134:131-6.

40. Knöchel C, Oertel-Knöchel V, Schönmeyer R, Rotarska-Jagiela A, Van de V V, Prvulovic D, et al. Interhemispheric hypoconnectivity in schizophrenia: fiber integrity and volume differences of the corpus callosum in patients and unaffected relatives. Neuroimage 2012;59:926-934.

41. Bai Y, Wang W, Xu J, Zhang F, Yu H, Luo C, et al. Altered resting-state regional homogeneity after 13 weeks of paliperidone injection treatment in schizophrenia patients. Psychiatry Res Neuroimaging. 2016;258:37-43. https://doi.org/10.1016/j.pscychresns.2016.10.008 Elsevier.

42. Lesh TA, Tanase C, Geib BR, Niendam TA, Yoon JH, Minzenberg MJ, et al. A multimodal analysis of antipsychotic effects on brain structure and function in first-episode schizophrenia. JAMA Psychiatry. 2015;72:226-34.

43. Siever LJ, Davis KL. The pathophysiology of schizophrenia disorders: perspectives from the Spectrum. Am J Psychiatry. 2004;161:398-413.

44. Cobia DJ, Smith MJ, Wang L, Csernansky JG. Longitudinal progression of frontal and temporal lobe changes in schizophrenia. Schizophr Res. 2012; 139:1-6.

45. Liu H, Liu Z, Liang M, Hao Y, Tan L, Kuang F, et al. Decreased regional homogeneity in schizophrenia: a resting state functional magnetic resonance imaging study. Neuroreport. 2006;17:19-22.

46. Guo W, Hu M, Fan X, Liu F, Wu R, Chen J, et al. Decreased gray matter volume in the left middle temporal gyrus as a candidate biomarker for schizophrenia: a study of drug naive, first-episode schizophrenia patients and unaffected siblings. Schizophr Res. 2014;159:43-50. https://doi.org/10. 1016/j.schres.2014.07.051 Elsevier B.V.

47. Goghari VM, MacDonald AW, Sponheim SR. Temporal lobe structures and facial emotion recognition in schizophrenia patients and nonpsychotic relatives. Schizophr Bull. 2011;37:1281-94.
Ready to submit your research? Choose BMC and benefit from:

- fast, convenient online submission

- thorough peer review by experienced researchers in your field

- rapid publication on acceptance

- support for research data, including large and complex data types

- gold Open Access which fosters wider collaboration and increased citations

- maximum visibility for your research: over $100 \mathrm{M}$ website views per year

At $\mathrm{BMC}$, research is always in progress.

Learn more biomedcentral.com/submissions 